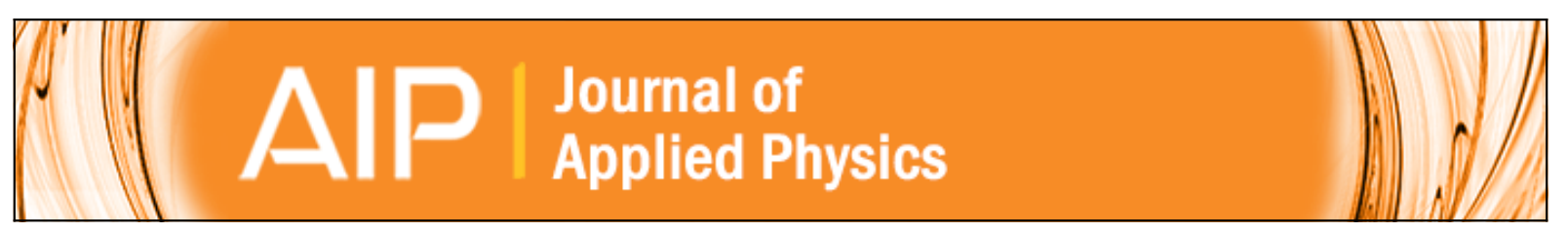

\title{
Electron paramagnetic resonance and Raman spectroscopy studies on carbon-doped
} MgB2 superconductor nanomaterials

Ali Bateni, Emre Erdem, Sergej Repp, Selcuk Acar, Ilkin Kokal, Wolfgang Häßler, Stefan Weber, and Mehmet Somer

Citation: Journal of Applied Physics 117, 153905 (2015); doi: 10.1063/1.4918608

View online: http://dx.doi.org/10.1063/1.4918608

View Table of Contents: http://scitation.aip.org/content/aip/journal/jap/117/15?ver=pdfcov

Published by the AIP Publishing

\section{Articles you may be interested in}

Al-doped MgB2 materials studied using electron paramagnetic resonance and Raman spectroscopy

Appl. Phys. Lett. 108, 202601 (2016); 10.1063/1.4949338

Doping effect of nano-Ho2O3 and naphthalene in MgB2 superconductor prepared by powder-in-sealed-tube method

J. Appl. Phys. 117, 113903 (2015); 10.1063/1.4915348

A new scaling relation for $\mathrm{n}$-AIN doped superconducting MgB2

J. Appl. Phys. 114, 093906 (2013); 10.1063/1.4820381

Effect of thermal strain on Jc and Tc in high density nano-SiC doped MgB2

J. Appl. Phys. 109, 07E108 (2011); 10.1063/1.3549590

Superconductivity and the disorder effect in Ag and Al double doped $\mathrm{Mg} \mathrm{B} 2$

J. Appl. Phys. 100, 023905 (2006); 10.1063/1.2219695

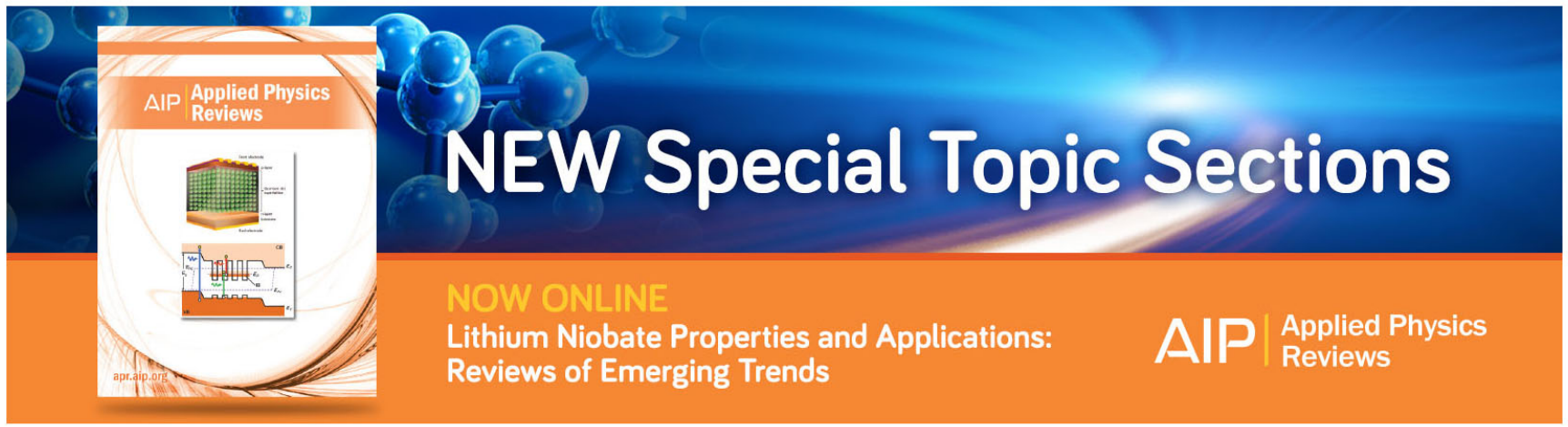




\title{
Electron paramagnetic resonance and Raman spectroscopy studies on carbon-doped $\mathrm{MgB}_{2}$ superconductor nanomaterials
}

\author{
Ali Bateni, ${ }^{1}$ Emre Erdem, ${ }^{2, a)}$ Sergej Repp, ${ }^{2}$ Selcuk Acar, ${ }^{3}$ Ilkin Kokal, ${ }^{3}$ Wolfgang Häßler, ${ }^{4}$ \\ Stefan Weber, ${ }^{2}$ and Mehmet Somer ${ }^{1, a)}$ \\ ${ }^{1}$ Department of Chemistry, Koc University, RumelifeneriYolu, Sariyer, Istanbul, Turkey \\ ${ }^{2}$ Institut für Physikalische Chemie, Universität Freiburg, Albertstr. 21, 79104 Freiburg, Germany \\ ${ }^{3}$ Pavezyum Kimya Sanayi Dlş Ticaret LTD. STTI., Tuzla, Istanbul, Turkey \\ ${ }^{4}$ Leibniz Institute for Solid State and Materials Research Dresden (IFW), P.O. Box 270116, 01171 Dresden, \\ Germany
}

(Received 10 March 2015; accepted 8 April 2015; published online 20 April 2015)

\begin{abstract}
Undoped and carbon-doped magnesium diboride $\left(\mathrm{MgB}_{2}\right)$ samples were synthesized using two sets of mixtures prepared from the precursors, amorphous nanoboron, and as-received amorphous carbon-doped nanoboron. The microscopic defect structures of carbon-doped $\mathrm{MgB}_{2}$ samples were systematically investigated using X-ray powder diffraction, Raman and electron paramagnetic resonance spectroscopy. $\mathrm{Mg}$ vacancies and C-related dangling-bond active centers could be distinguished, and $\mathrm{sp}^{3}$-hybridized carbon radicals were detected. A strong reduction in the critical temperature $T_{\mathrm{c}}$ was observed due to defects and crystal distortion. The symmetry effect of the latter is also reflected on the vibrational modes in the Raman spectra. (C) 2015 AIP Publishing LLC.
\end{abstract}

[http://dx.doi.org/10.1063/1.4918608]

\section{INTRODUCTION}

The relatively low density $\left(\rho=2.55 \mathrm{~g} / \mathrm{cm}^{3}\right),{ }^{1}$ high critical temperature $\left(T_{\mathrm{c}} \approx 39 \mathrm{~K}\right){ }^{2}{ }^{2}$ simple crystal structure, and chemical composition are outstanding properties of magnesium diboride $\left(\mathrm{MgB}_{2}\right)$ that lead to promising applications in modern engineering. $\mathrm{MgB}_{2}$ materials are nowadays widely been employed, e.g., in magnetic-resonance imaging (MRI) magnets operating at cryogenic temperatures, ${ }^{3-6}$ maglev transport, ${ }^{7,8}$ power transmission lines, ${ }^{9,10}$ high-sensitivity and low-voltage sensors, ${ }^{11}$ low-cost and high-efficiency offshore wind turbines for clean energy future, ${ }^{12}$ and other renewable energy devices such as 2 nd generation superconductor wires and coils. ${ }^{10,13}$ Very recently, a world-record electric-current flow (more than $3 \mathrm{MA}$ ) from a power converter to magnets has been achieved in CERN's LHC particle accelerator by using $\mathrm{MgB}_{2}$ transmission cables. ${ }^{14} \mathrm{MgB}_{2}$ is also a promising candidate material for a feedback coil, which is an important component for plasma confinement. ${ }^{15}$ Details on practical applications of $\mathrm{MgB}_{2}$-based wires can be found in a recent review article. ${ }^{4}$ Such applications make use of the two-band superconducting properties of $\mathrm{MgB}_{2}$, which lead to relatively high values of the critical current density $\left(J_{\mathrm{c}}\right)$ and critical magnetic field $\left(H_{\mathrm{c}}\right)$. Since these two parameters determine the basic superconducting characteristics of $\mathrm{MgB}_{2}$, they have been mostly addressed in recent research. Studies on $J_{\mathrm{c}}, H_{\mathrm{c}}$, and $T_{\mathrm{c}}$ have been discussed in quite some detail in a number of comprehensive reviews. ${ }^{1,16,17}$ However, $J_{\mathrm{c}}$ and the upper critical field $\left(H_{\mathrm{c} 2}\right)$ in $\mathrm{MgB}_{2}$ are targets for further improvement. ${ }^{7}$ Introducing disorder by doping and/or exploiting the existence of intrinsic defects in the lattice structure of $\mathrm{MgB}_{2}{ }^{7,18-20}$ may lead to even more favorable superconductive properties.

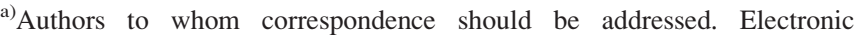
addresses: emre.erdem@physchem.uni-freiburg.de and msomer@ku.edu.tr
}

The performance of $\mathrm{MgB}_{2}$ as a superconductor is strongly influenced by the choice of starting materials; especially the degree of crystallinity and the particle size of the boron precursor are of utmost importance. ${ }^{21}$ The main difficulty in preparing $\mathrm{MgB}_{2}$ from crystalline boron is its inert reaction with $\mathrm{Mg}$ powder to form $\mathrm{MgB}_{2}$. This is mainly due to the thermodynamic stability of the $\beta$-rhombohedral crystal modification and the high Mohs hardness of crystalline boron (9.5). Thus, production of $\mathrm{MgB}_{2}$ using crystalline boron requires long sintering times and quite high temperatures. ${ }^{4}$ Enhanced reactivity of the boron precursor can be achieved by reducing the particle size to nano-dimensions and using the amorphous boron modification. ${ }^{4}$ Recently, we could confirm that the quite high content of intrinsic defects in amorphous nanoboron, as supplied by the company Pavezyum, yields $\mathrm{MgB}_{2}$ material with unique and superior characteristics. ${ }^{22}$ Although various $\mathrm{MgB}_{2}$ compounds have been extensively investigated over the past decades, there is still considerable controversy about of its various properties. In particular, the defect structures of undoped and doped $\mathrm{MgB}_{2}$ have remained unclear, especially when the crystallite or particle size is confined to nanometer dimensions. We previously reported that a significant reduction of the average particle size of $\mathrm{MgB}_{2}$ can be achieved by using amorphous nanoboron precursors. ${ }^{22}$ The origin and the role of defect centers were also identified: at bulk-scale and at nano-scale, undoped $\mathrm{MgB}_{2}$ material contains plenty of $\mathrm{O}-$ and $\mathrm{Mg}$ vacancies, respectively. ${ }^{22}$

Although the various compound morphologies play a decisive role to control its properties, the synthesis method and doping define additional, crucial parameters that markedly impact the nature and concentration of intrinsic or extrinsic defects and the materials' electronic properties. To this end, it has been reported that Carbon (C) doping significantly improves the electromagnetic properties ${ }^{3}$ and the critical 
current density ${ }^{18,23}$ of $\mathrm{MgB}_{2}$. Furthermore, it is also known, that adding of single wall $\mathrm{C}$ nanotubes and nano-SiC in $\mathrm{MgB}_{2}$ enhance the flux pining, thus the superconducting performance. ${ }^{24,25}$ Some other additives such as nano- $\mathrm{Co}_{3} \mathrm{O}_{4}$ can also act as effective pining centers, and hence increase the $J_{\mathrm{c}}$, being coupled with fluxoid jumps. ${ }^{26}$ Nonetheless, the effect of $\mathrm{C}$ doping in $\mathrm{MgB}_{2}$ has also been discussed controversially with respect to its substitution site and solubility limit. ${ }^{18,23,27,28}$ Moreover, previous studies on $\mathrm{C}$ doping into $\mathrm{MgB}_{2}$ were mainly focused on its effect on superconductivity. From the defect structure point of view, the effect of $\mathrm{C}$ doping on the electronic properties is equally important and has to be investigated. In this work, the defect structure of C-doped $\mathrm{MgB}_{2}$, and its impact on the material properties will be investigated by electron paramagnetic resonance (EPR) and Raman spectroscopy. EPR is well suited for this task because it provides a direct tool to monitor different paramagnetic states of defects and, thus, complements other experimental techniques such as X-ray powder diffraction (XRPD) and Raman spectroscopy. In this sense, EPR does not only allow to identify paramagnetically active intrinsic and extrinsic defects but also yielding insight into the correlation of such defects to the related structural and superconductive properties of $\mathrm{MgB}_{2}$. Moreover, by using spin counting, EPR enables us to determine analytically the absolute concentration of defect centers and/or doping ions, here C. Since $\mathrm{MgB}_{2}$ is a phonon-mediated superconductor, electronphonon interaction plays an important role in the pairing mechanism that is responsible for $\mathrm{MgB}_{2}$ superconductivity. ${ }^{29,30}$ However, unclear issues such as phonon anomalies like the anomalously large linewidth of the $E_{2 g}$ phonon mode, the anharmonicity, and multi-phonon contributions may be closely relate with the defects. ${ }^{31,32}$ The importance of phonon-defect interaction as an alternative cause for the anomalies of high-temperature superconductors have been emphasized in earlier reports. ${ }^{33}$ Therefore, the correlation of Raman and EPR results would give plausible information about the defect related electron-phonon or phonon-defect interactions, which most likely contribute to the Raman and EPR spectra of undoped and C-doped $\mathrm{MgB}_{2}$.

\section{EXPERIMENTAL}

\section{A. Synthesis of carbon-doped $\mathrm{MgB}_{2}$ powders}

Solid-state synthesis was chosen for all sample preparations. Two sets of mixtures with different boron precursors have been used as starting materials. To distinguish between different boron precursors, amorphous nanoboron and asreceived amorphous $\mathrm{C}$-doped nanoboron were labeled as $\mathrm{P} 1$ and $\mathrm{P} 2$, respectively.

Set1: One set of undoped and C-doped P1 mixtures were prepared using starting powders of the following purity grades: $\mathrm{Mg}$ (99.8 wt.\%, Pavezyum), amorphous nanoboron (B > 98.5 wt.\%, Pavezyum), DL-malic acid (MA) $\left(\mathrm{C}_{4} \mathrm{H}_{6} \mathrm{O}_{5}\right.$ (99\%, Merck)). Systematic C doping was performed on all powders by using DL-malic acid as a $\mathrm{C}$ source with a method reported previously. ${ }^{34} \mathrm{P} 1$ is mixed with $0,0.1,1,5,10$, and $30 \mathrm{wt}$ \% of MA in a toluene medium for $1 \mathrm{~h}$. Subsequently, toluene was evaporated at $150^{\circ} \mathrm{C}$ for $3 \mathrm{~h}$ to remove all volatile organics.

Set2: Another set of undoped and C-doped P2 blends were prepared using starting powders of following purity grades: $\mathrm{Mg}$ (99.8 wt.\%, Pavezyum), as-received C-doped amorphous nanoboron with 1.85 wt. \% ( 2 mol. \%) C concentration (Pavezyum).

In all series, $\mathrm{Mg}$ - and $\mathrm{C}$-doped precursors (P1 and P2) were placed in a stoichiometric ratio into diamagnetic zirconia air-tight milling vials in a glove box and mixed at $500 \mathrm{rpm}$ using a Fritsch P7 planetary ball mill for $30 \mathrm{~min}$. Afterwards, pellets of $13 \mathrm{~mm}$ diameter were pressed with a hydraulic press employing a pressure of $10 \mathrm{t} / \mathrm{cm}^{2}$. The samples were put into small iron crucibles and placed in an iron reactor, which was purged by Ar gas during the heating process to avoid oxidation. The details of the synthesis procedure are as follows: $2 \mathrm{~h}$ fast heating to close to the melting point of magnesium $\left(25-600^{\circ} \mathrm{C}\right)$ and slow heating to minimize $\mathrm{Mg}$ evaporation for $5 \mathrm{~h}$ up to $800^{\circ} \mathrm{C}\left(600-800^{\circ} \mathrm{C}\right)$ and allowing to cool down in the furnace.

$\mathrm{MgB}_{2}$ samples produced from Set1 were labeled according MA concentration (wt. \%): 0CS1 (0 wt. \%), 0.1CS1 (0.1 wt. \%), 1CS1 (1 wt. \%), 5CS1 (5 wt. \%), 10CS1 (10 wt. \%), and 30CS1 (30 wt. \%). Two $\mathrm{MgB}_{2}$ samples synthesized by using Set2 were coded based on C concentration (wt. \%) as 0CS2 $(0$ wt. \%) and $1.85 \mathrm{CS} 2(1.85$ wt. \%) for undoped and 1.85 wt. \% ( 2 mol. \%) C-doped samples, respectively (Table I.).

\section{B. Methods of sample characterisation}

All samples were analyzed by XRPD. XRPDs were recorded with a BRUKER D2 Phaser diffractometer $(\mathrm{Cu}$ radiation with LYNXEYETM detector). Rietveld refinement was carried out on all samples for phase characterization and determination of the lattice parameters $a$ and $c$ by using the TOPAS software. CIF files for Raman analysis were done using nearly $5 \mathrm{mg}$ of sample in sealed Pyrex tube $(\varnothing=4 \mathrm{~mm})$ in the range of $2500-1000 \mathrm{~cm}^{-1}$ with a BURKER RFS $100 / \mathrm{S}$ spectrometer (Nd:YAG-Laser, $1064 \mathrm{~nm}, 200 \mathrm{~mW}$ ). X-band $(9.86 \mathrm{GHz})$ continuous-wave-EPR measurements were performed with a Bruker EMX spectrometer. We have used a rectangular TE102 (X-band) resonator from Bruker. The offset in the magnetic field and the exact $g$-factors in X-band measurements were determined with a polycrystalline DPPH (2-diphenyl-1-picrylhydrazyl) reference sample with well-

TABLE I. Sample codes and corresponding wt. \% values for the doping concentration.

\begin{tabular}{lc}
\hline \hline Sample name & wt. \% of added dopants \\
\hline $0 \mathrm{CS} 1$ & 0 \\
$0.1 \mathrm{CS} 1$ & 0.1 \\
$1 \mathrm{CS} 1$ & 1 \\
$5 \mathrm{CS} 1$ & 5 \\
$10 \mathrm{CS} 1$ & 10 \\
$30 \mathrm{CS} 1$ & 30 \\
$0 \mathrm{CS} 2$ & 0 \\
$1.85 \mathrm{CS} 2$ & 1.85 \\
\hline \hline
\end{tabular}



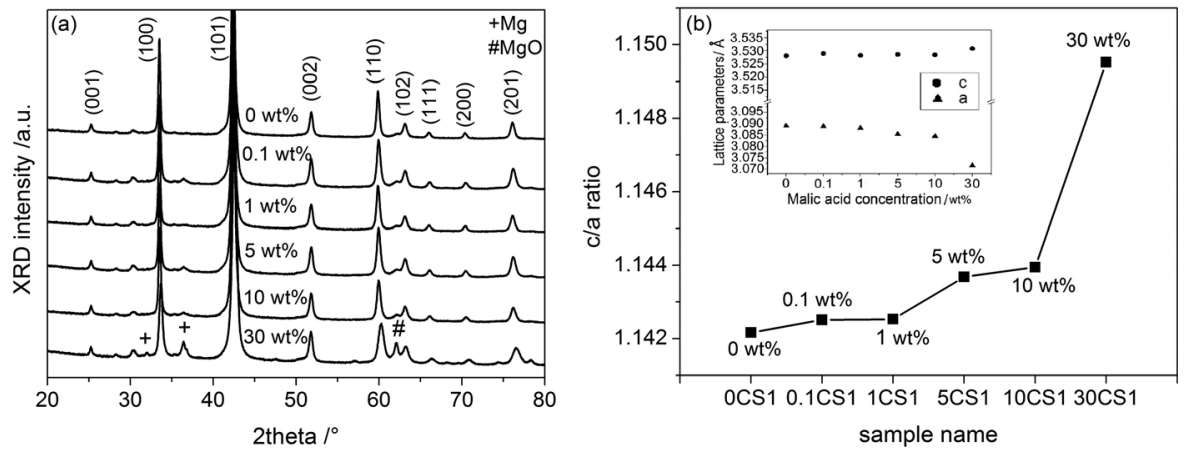

FIG. 1. (a) XRPD patterns, (b) $c / a$ ratio and lattice parameters $a$ and $c$ (inset), which were obtained from a Rietveld refinement analysis of set1 samples (set1) with $0,0.1,1,5,10$, 30 wt. $\%$ of MA. known $g$-factor $(g=2.0036)$. For cooling (to liquid helium temperatures) an Oxford CF-935 cryostat was used. The temperature was regulated by a temperature controller (Oxford ITC-503). The EPR spectral analysis has been performed using the WINEPR program from Bruker.

\section{RESULTS AND DISCUSSION}

\section{A. Structural analysis}

XRPD results of $\mathrm{MgB}_{2}$ synthesized from set1 samples with $0,0.1,1,5,10$, and $30 \mathrm{wt}$. $\%$ of MA are plotted in Fig. 1(a). $\mathrm{MgB}_{2}$ (ICSD Collection Code: 93925) is the main product but small amounts of $\mathrm{MgO}$ (ICSD Collection Code: 26958) are also detected in all samples. An excess amount of $\mathrm{Mg}$ is observed at higher MA concentrations in C-doped $\mathrm{MgB}_{2}$ samples due to restricted availability of boron. To learn more on the effect of $\mathrm{C}$ doping into the $\mathrm{MgB}_{2}$ structure, a Rietveld analysis was performed on all synthesized samples and the $c / a$ ratio with the lattice parameters $a$ and $c$ (in the inset) are plotted in Fig. 1(b). With rising MA content, the $c / a$ ratio increases steadily along with a decrease in $a$, while $c$ remains nearly unchanged except for the highest MA concentration. The contraction of the lattice parameter $a$ and the accompanying increase of the $c / a$ ratio is attributed to progressing substitution of $\mathrm{C}$ (atomic radius $0.77 \AA$ ) into boron sites with (atomic radius $0.97 \AA$ ). ${ }^{23,35-37}$

The XRPD patterns of $\mathrm{MgB}_{2}$ synthesized from set2 samples have been published elsewhere. ${ }^{22}$ They showed similar results as the set1 series with $\mathrm{MgB}_{2}$ as the main product. Lattice parameters $a$ and $c$ of undoped and $1.85 \mathrm{wt} \%$ (2 mol. \%) C-doped samples were calculated as 3.0888(5) and 3.5281(3) $\AA$, and 3.0828(7) $\AA$ and 3.5274(1) $\AA$, respectively. It should be noted that for $\mathrm{C}$ doping into $\mathrm{MgB}_{2}$, the carbons can either substitute into boron sites or into interstitial sites within boron rings and grain boundaries. ${ }^{23,38}$ The very small increase in lattice parameter $c$ shown in Fig. 1(b) may be related to interstitial $\mathrm{C}$ substitution into $\mathrm{MgB}_{2} \cdot{ }^{11,23}$ Therefore, there is a difference between the real $\mathrm{C}$ doping into $\mathrm{B}$ sites and the nominal $\mathrm{C}$ concentration, which is the total amount of added C, and the terms "real" and "nominal" are used in the literature to differentiate between them..$^{27,28,34,39}$ In the literature, various methods can be found to calculate the real $\mathrm{C}$ concentration. The real $\mathrm{C}$ concentration in $\operatorname{Mg}\left(\mathrm{C}_{x} \mathrm{~B}_{1-x}\right)_{2}$ can be calculated from the semi-empirical formula $x=7.5 \Delta(c / a)$, in which $\Delta(c / a)$ is the difference between the $c / a$ ratio of C-doped samples and the $c / a$ ratio of undoped sample
$\left(\Delta(c / a)=(c / a)_{\text {undoped }}-(c / a)_{\text {doped }}\right) .^{40} \mathrm{MA}$, nominal, and real $\mathrm{C}$ doping concentration in $\mathrm{MgB}_{2}$ are compiled in Table II.

From Table II, it can be seen for sample $0.1 \mathrm{CS} 1$, that the real $\mathrm{C}$ doping amount is exceeding the nominal $\mathrm{C}$-doped concentration. This may be related to the formation of $\mathrm{MgO}$, which is reducing the $\mathrm{Mg}$ amount relative to $\mathrm{C}$-doped concentration. $^{28,39}$

\section{B. Spectroscopic analysis}

\section{Raman spectroscopy}

The Raman modes of $\mathrm{MgB}_{2}$, and in particular, those of the C-doped ones, have not been fully understood for a very long time. ${ }^{31,41-43}$ Specifically, the background signal and the broad linewidth of the soft mode ( $E_{2 g}$ phonon mode) were rendering the Raman interpretation quite difficult. Clearly, $\mathrm{MgB}_{2}$ represents a two-gap superconductor with the Fermi surface consisting of two-dimensional $\sigma$-bands and threedimensional $\pi$-bands. Group theory predicts for the $P 6 / \mathrm{mmm}$ space group in $\mathrm{MgB}_{2}$ the following four vibration modes at the Brillouin zone: a silent $B_{1 g}$ mode, the $E_{2 g}$ Raman mode, and the infrared-active $\mathrm{u}$ modes $E_{1 u}$ and $A_{2 u}$. Among them only the $E_{2 g}$ mode (soft mode) is Raman active. This mode mainly results from the strong coupling between electrons and phonons and is located around $590-660 \mathrm{~cm}^{-1}$ with the wide linewidth $\left(\sim 200 \mathrm{~cm}^{-1}\right){ }^{41-45}$ The strong interaction of the $E_{2 g}$ phonon with the $\sigma$ electronic bands is believed to be the main contribution to superconductivity. ${ }^{43}$ Motaman et al. reported also that the $E_{2 g}$ phonon mode is related to the inplane vibration mode of the boron atom. ${ }^{42}$ Therefore, the $E_{2 g}$ phonon mode can be easily influenced by lattice distortion (defects) or atomic substitution (doping).

TABLE II. MA, nominal $\mathrm{C}$, and real $\mathrm{C}$ doping concentrations in $\mathrm{MgB}_{2}$.

\begin{tabular}{lccc}
\hline \hline $\begin{array}{l}\text { Sample } \\
\text { name }\end{array}$ & $\begin{array}{c}\text { Dopant } \\
\text { concentration } \\
(\text { wt. } \%)\end{array}$ & $\begin{array}{c}\text { Nominal C } \\
\text { concentration } \\
x \times 10^{-2}(\mathrm{~mol} . \%)\end{array}$ & $\begin{array}{c}\text { Real C } \\
\text { concentration } \\
x \times 10^{-2}(\mathrm{~mol} \%)\end{array}$ \\
\hline 0CS1 & 0 & 0 & 0 \\
$0.1 \mathrm{CS} 1$ & 0.1 & 0.036 & 0.25 \\
$1 \mathrm{CS} 1$ & 1.0 & 0.360 & 0.276 \\
$5 \mathrm{CS} 1$ & 5.0 & 1.790 & 1.140 \\
$10 \mathrm{CS} 1$ & 10.0 & 3.580 & 1.340 \\
$30 \mathrm{CS} 1$ & 30.0 & 10.700 & 5.520 \\
$0 \mathrm{CS} 2$ & 0 & 0 & 0 \\
$1.85 \mathrm{CS} 2$ & 1.85 & 2.0 & 1.530 \\
\hline \hline
\end{tabular}


In this study, we have measured Raman spectra of nanosized samples of pure $\mathrm{MgB}_{2}$, as well as of doped ones with $0,0.1$, and $10 \mathrm{wt}$. \% of MA (named as: 0CS1, 0.1CS1, and 10CS1, respectively), to examine the $\mathrm{C}$ doping effects on the phonon modes. In Fig. 2(a), the most prominent boron-atomrelated $E_{2 g}$ phonon mode was observed at $573 \mathrm{~cm}^{-1}$, however, it appeared at slightly lower wavenumbers than in bulk $\mathrm{MgB}_{2}\left(<590-660 \mathrm{~cm}^{-1}\right.$ band). This shift results from the unique property of nanoboron ${ }^{22}$ due to size effect, which is used as precursor for $\mathrm{MgB}_{2}$ syntheses. The main effect of nanoboron as starting material is the increase of the concentration of $\mathrm{Mg}$ vacancies as defect centers in $\mathrm{MgB}_{2}$. Therefore, the defect scattering and band filling reduces the density of states and alters the shape of the Fermi surface. The filling of the vacancies by $\mathrm{C}$ doping is expected to affect the electronic screening of $\mathrm{B}$ and $\mathrm{Mg}$ atom displacements and changing the interatomic distances and forces between the atoms in $\mathrm{MgB}_{2}$. Such an effect has been observed recently for Al-doped $\mathrm{MgB}_{2}$ materials. ${ }^{32}$ The decrease (red shift) in phonon-mode energy in nanoboron $\mathrm{MgB}_{2}$ sample can be mainly attributed to the decreased density of states. As depicted in Fig. 2(b), the $E_{2 g}$ phonon peak shifts to the lower energy side with $0.1 \mathrm{wt}$. \% of MA with respect to undoped $\mathrm{MgB}_{2}$ and shifts further very slightly to the lower energy side with 10 wt. \% of MA (refer Fig. 2(c)). The two additional peaks around 745 and $1027 \mathrm{~cm}^{-1}$ are only visible in C-doped samples indicating their strong relationship with the doping effect, i.e., $\mathrm{C}$ addition. Both modes show red shifts with increasing amount of MA. The decrease in the intensity of the $E_{2 g}$ phonon mode has been reported by several groups and is also attributed to local symmetry breaking due to substitution effects, hence C-related defects. ${ }^{31,43,46}$ In particular, the Raman mode at $745 \mathrm{~cm}^{-1}$ has been reported earlier and explicitly assigned to the phonon density of states due to disorder. ${ }^{46}$ Both additional peaks may not be described by $P 6 / \mathrm{mmm}$ space group and need advanced computational modeling. ${ }^{31}$

\section{EPR spectroscopy}

An adequate spin Hamiltonian for $S=1 / 2$ electron-spin centers interacting with a nucleus of arbitrary nuclear spin $I$ is given by
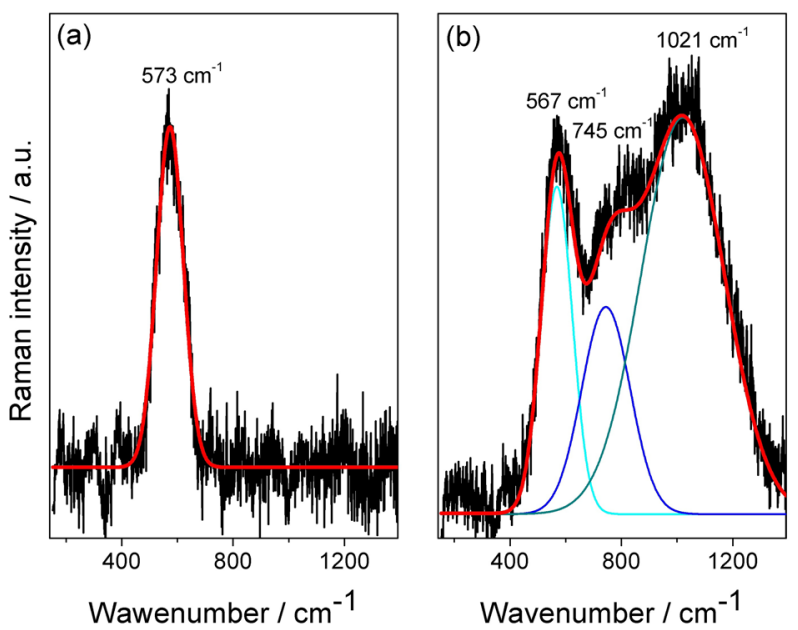

$$
H=\mathbf{g}_{e} \beta_{e} \mathbf{B}_{0} \cdot \mathbf{S}-g_{n} \beta_{n} \mathbf{B}_{0} \cdot \mathbf{I}+\mathbf{S} \cdot \mathbf{A} \cdot \mathbf{I} .
$$

The nuclear $g$-factor, and the electron and nuclear Bohr magnetons are denoted $g_{\mathrm{n}}, \beta_{\mathrm{e}}$, and $\beta_{\mathrm{n}}$, respectively. The first two terms describe the electronic and nuclear Zeeman interactions, and the last term specifies the hyperfine interaction to adjacent magnetic nuclei. $B_{0}$ describes the external field strength. The nuclear quadrupole interaction to nuclei with $I \geq 1$ has been neglected. As a finger-print type information, in $\mathrm{MgB}_{2}$ the hyperfine interaction to different magnetically active nuclei has to be considered. There are three magnesium isotopes, where ${ }^{24} \mathrm{Mg}$ and ${ }^{26} \mathrm{Mg}(I=0$, with natural abundance of $78 \%$ and $11 \%$, respectively) are the most abundant ones and ${ }^{25} \mathrm{Mg}$ is usually neglected $(I=5 / 2$ at $10 \%$ natural abundance). Boron exists as two magnetically active isotopes, where ${ }^{11} \mathrm{~B}(I=3 / 2$ and $80.1 \%$ natural abundance) is the dominant one and the isotope ${ }^{10} \mathrm{~B}$ is again typically neglected $(I=3$ and $19.9 \%$ natural abundance). Concerning $\mathrm{C}$, the most abundant isotope,${ }^{12} \mathrm{C}$, has vanishing nuclear spin $(I=0)$, and only the ${ }^{13} \mathrm{C}$ isotope $(I=1 / 2$ and $1.11 \%$ natural abundance) bears a nuclear spin. Hyperfine interactions with ${ }^{1} \mathrm{H}$ nuclei $(I=1 / 2)$ remain typically unresolved in the EPR spectra and lead only to an inhomogeneous line broadening. All measured EPR signals exhibit no hyperfine structure, hence, a localization of the unpaired electron spin on magnesium ${ }^{25} \mathrm{Mg}(I=5 / 2)$ or boron ${ }^{11} \mathrm{~B}(I=3 / 2)$ can be ruled out. Instead, the observed EPR signals in Fig. 3(a) can be assigned to either paramagnetic C-based "dangling bond" (DB) species ${ }^{47}$ or to intrinsic defects, such as $\mathrm{Mg}$ vacancies. ${ }^{22}$ For an assignment of the observed DB centers to either C- or Mg-vacancy species, the $g$-values as determined from EPR data can be used. C radical centers typically occur in an interval of $g$-values ranging from $g_{\mathrm{C}} \sim 2.0020-2.0030 .{ }^{48-52}$ In contrast, Mg-vacancy centers in nanosized $\mathrm{MgB}_{2}$ typically exhibit $g$-values around 2.0017. ${ }^{22}$ Obviously, as presented in Fig. 3(b), the observed EPR signals of C-doped $\mathrm{MgB}_{2}$ can be assigned to $\mathrm{C}$ "dangling bonds" whereas the undoped signal to $\mathrm{Mg}$-vacancies. Moreover, from the EPR data it may be possible to distinguish between $\mathrm{sp}^{2}$ - and $\mathrm{sp}^{3}$-hybridization by considering the EPR spectral linewidth $\left(\Delta B_{0}\right)$. Values for $\mathrm{sp}^{3}$-hybridized $\mathrm{C}$ centers, as found in diamond for

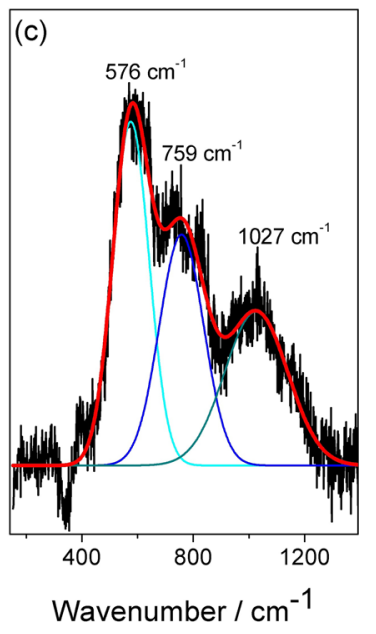

FIG. 2. Raman spectra of samples (set1) with (a) 0 wt. \%, (b) 0.1 wt. $\%$, and (c) 10 wt. \% of MA. 


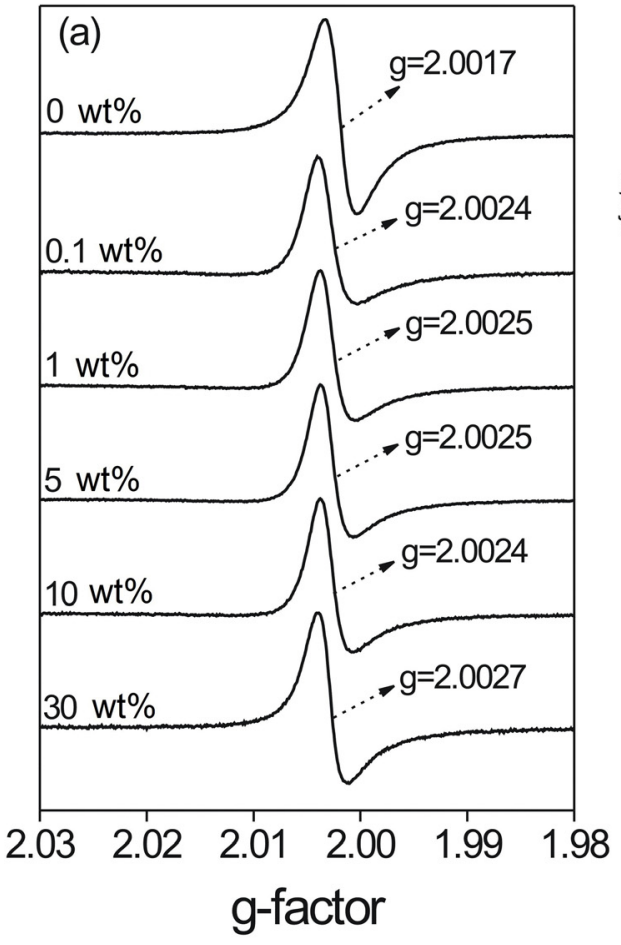

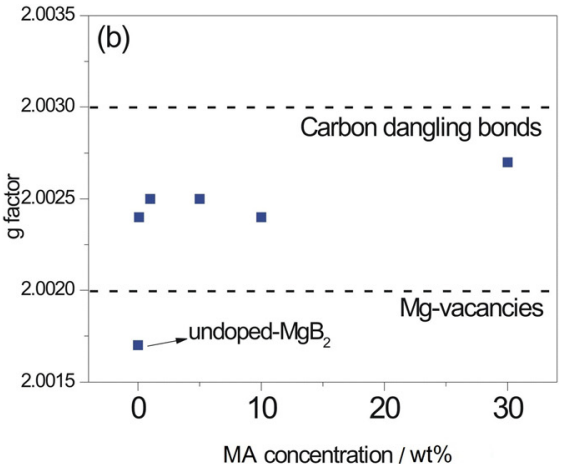

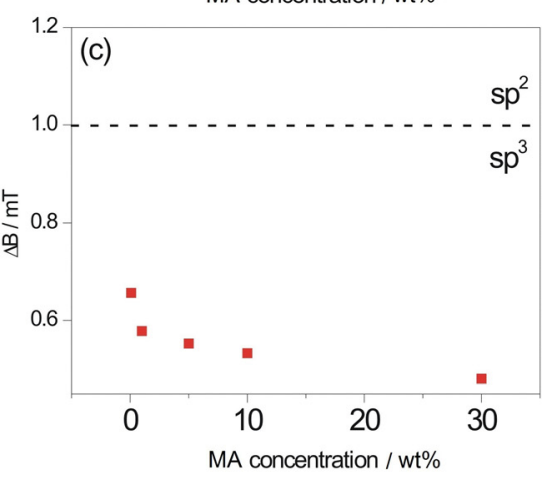

FIG. 3. (a) Room-temperature EPR spectra (first derivatives with respect to $B_{0}$ ) of undoped $\mathrm{MgB}_{2}$ and doped $\mathrm{MgB}_{2}$ with various $\mathrm{C}$ doping levels (with $0,0.1,1,5,10$, and 30 wt. $\%$ of MA). (b) Electron $g$-factors of $\mathrm{MgB}_{2}$ both for doped and undoped case determined from the inflection points of the spectra. The corresponding regions for $\mathrm{C}$ related and vacancy related centers are indicated. ${ }^{22,48-52}$ (c) EPR linewidths of C-doped $\mathrm{MgB}_{2}$. example, have been determined to $\Delta B_{\mathrm{sp} 3} \leq 1 \mathrm{mT} .^{47,51}$ For graphite-like $\mathrm{C}$ centers with $\mathrm{sp}^{2}$-hybridization, generally broader EPR linewidths, $\Delta B_{\mathrm{sp} 2} \geq 1 \mathrm{mT}$, are observed. ${ }^{47,51}$ The corresponding variation in $\Delta B$ is represented in Fig. 3(c), and we clearly distinguish, experimentally, the difficult issue of $\mathrm{C}$ hybridization state. The results strongly suggest predominantly $\mathrm{sp}^{3}$-hybridization to occur. This finding is corroborated based on theory by first principle studies. $^{53}$

The concentration of defect centers can also be determined by EPR. For this purpose, the first-derivative EPR signal is doubly integrated. By comparing the double integral with that of a standard sample of known spin concentration (here, $\mathrm{MnO}$ powder with $1.495 \times 10^{18}$ spins), one obtains the corresponding number of spins in the sample under investigation, and thus, the concentration of defect centers. ${ }^{54,55}$ Here, we have specifically chosen the $1.85 \mathrm{wt}$. \% (2 mol. \%) C-doped sample synthesized from the set 2 blend. Spin counting resulted in $1.3 \times 10^{18}$ spins per $\mathrm{mg}$ sample, which corresponds to $1.45 \mathrm{~mol}$. \% C concentration. This is quite consistent with the value $1.53 \mathrm{~mol}$. \% $\mathrm{C}$ obtained from the Rietveld refinement analysis (cf. Table II). Both EPR and Rietveld results suggest that a small amount of $\mathrm{C}$ either remains in a diamagnetic state or is not incorporated into the $\mathrm{MgB}_{2}$ lattice but rather distributed around and/or on the grain boundaries. ${ }^{23,27,56}$

Figs. 4(a)-4(c) show X-band EPR spectra recorded in the temperature range between 40 and $10 \mathrm{~K}$. This range is important since it includes the critical temperature of $\mathrm{MgB}_{2}$. Therefore, it is quite important to compare EPR results of undoped and $\mathrm{C}$-doped $\mathrm{MgB}_{2}$ at low temperatures. In the former material, the EPR signal is induced by $\mathrm{Mg}$-vacancies whereas in the latter $\mathrm{C}$-dangling bonds give rise to the intense EPR signal at $g \sim 2$. Due to the different driving forces, one can expect unequal temperature-dependent EPR responses from undoped and $\mathrm{C}$-doped $\mathrm{MgB}_{2}$. The temperature dependence of $1 /(I \times T)$ falls off drastically around 25-30 K for undoped and C-doped $\mathrm{MgB}_{2}$. The EPR intensity
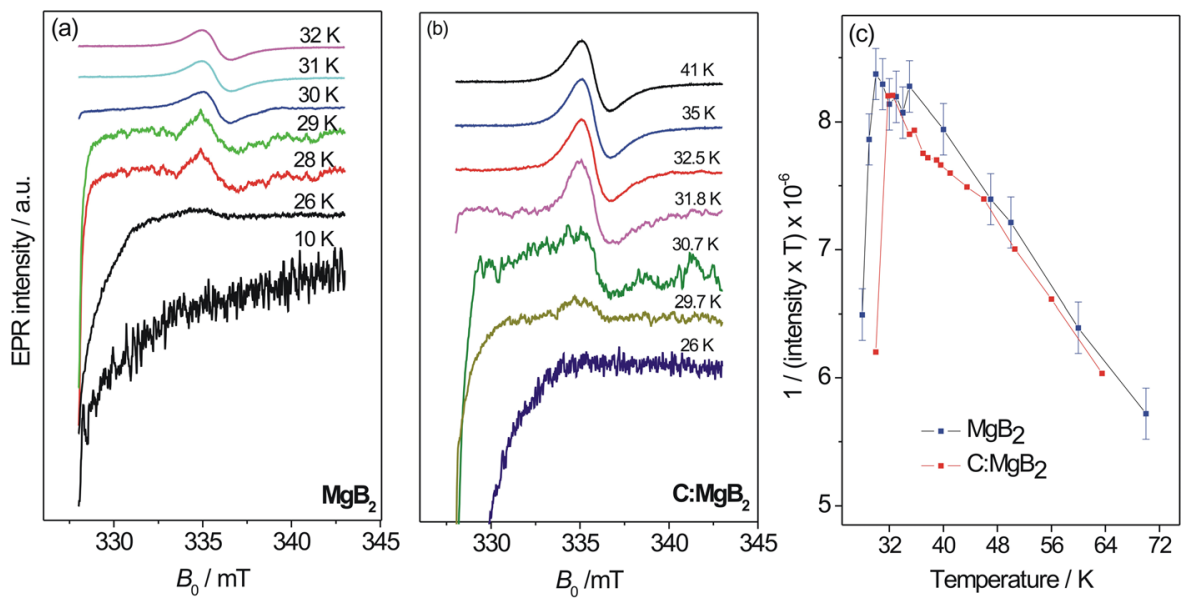

FIG. 4. Low-temperature EPR measurements (a) for undoped and (b) for nominally $1.85 \mathrm{wt}$. \% (2 mol. \%) Cdoped $\mathrm{MgB}_{2}$ synthesized from set2. (c) Comparison of temperature-dependent EPR signal for both undoped and $\mathrm{C}$ doped $\mathrm{MgB}_{2}$ material. 
is expected to be proportional to the dc susceptibility of the spins as can be seen in Fig. 4(c). Clearly, both samples follow the Curie-Weiss law $(\chi=C /(T-\theta)$, where $C$ and $\theta$ are the Curie constant and the Curie-Weiss temperature, respectively) thus indicating pure paramagnetic behavior until the respective critical temperature is reached. Similar CurieWeiss paramagnetism has been observed previously for $\mathrm{MgB}_{2}$ indirectly by SQUID measurements. ${ }^{57}$ Contrarily, it is of course not possible from EPR spectra to determine quantitatively the dependency of the superconducting fraction. ${ }^{58}$ Below the critical temperature, the susceptibility starts to strongly deviate from a linear temperature dependency, which means also deviation from the Curie-Weiss behavior. That shows that the spin dynamic properties of the samples are highly sensitive to the temperature, here $T_{\mathrm{c}}$. If the EPR intensity follows the Curie-Weiss law at low temperatures, this evidently enables us to assume that the Mg-vacancies and $\mathrm{C}$-related defects were both uniformly distributed in $\mathrm{MgB}_{2}$ samples. Below $T_{\mathrm{c}}$, paramagnetic defects in $\mathrm{MgB}_{2}$ completely smeared out and the materials become superconductive. However, just above $T_{\mathrm{c}}$, before the paramagnetic defects smeared out, both paramagnetically active defects $\left(\mathrm{V}_{\mathrm{Mg}}\right.$ or $\mathrm{C}$ radicals) still make exchange interactions with magnetic field and phonons thus cause quite instable EPR spectra. This can be seen in Figs. 4(a) and 4(b) clearly that, by approaching the $T_{\mathrm{c}}$ value the signal-to-noise ratio of EPR spectra becomes worse compared to higher temperatures. In particular, the lineshape of undoped $\mathrm{MgB}_{2}$, especially for EPR spectra measured at 29, 28, and $26 \mathrm{~K}$ (Fig. 4(a)) differs substantially from a pure Lorentzian, predominantly as a result of the inhomogeneous distribution of the magnetic field inside the material, caused by the appearance of the vortices. Such an EPR signal at the superconducting state was described recently by the realization of vortex structure in $\mathrm{MgB}_{2}$ samples. This effect has been also reported for different impurity centers in $\mathrm{MgB}_{2}{ }^{59} \mathrm{Fig}$. 4(c) shows that the superconducting state starts in the temperature region $25-30 \mathrm{~K}$. That means that defect-induced paramagnetism, quite interestingly, still holds even below the bulk $T_{\mathrm{c}}$ value (34 K). The decrease in $T_{\mathrm{c}}$ has been also reported for increasing level of $\mathrm{C}$ and rare-earth oxide doped $\mathrm{MgB}_{2}{ }^{60}$ This can be explained by three ways: (i) $\mathrm{MgB}_{2}$ synthesized by nanoboron precursor reduce the $T_{\mathrm{c}}$ due to its unique properties, (ii) interaction defects between phonons or between each other alter the superconducting temperature and/or, and (iii) defects or C doping enhances the lattice distortion. Moreover, these EPR results are highly in agreement with the above Raman spectroscopy results. Lowering of $T_{\mathrm{c}}$ found in this work from EPR data has been also reported from Raman studies and was identified with the disorder due to chemical substitution, crystal lattice contraction and electron doping induced electronic structure variation. This shows close correlation between the defect centers and vibration phonon modes, and hence, the superconductivity of $\mathrm{MgB}_{2}$.

\section{CONCLUSIONS}

In this work, $\mathrm{MgB}_{2}$ was synthesized as nanomaterial by a solid-state synthesis route. Importantly, fully amorphous nanoboron transfers its unique character to the nano-sized undoped and C-doped $\mathrm{MgB}_{2}$. The real $\mathrm{C}$ doping levels in $\mathrm{MgB}_{2}$ samples were determined using the XRPD and the Rietveld refinement data. The results were confirmed by EPR (quantitative spin counting). Both methods reveal reasonable agreement with each other. So far, the defect structure has not been taken into account for $\mathrm{MgB}_{2}$ superconductor and tremendous work has been published for understanding macroscopically the superconductivity in this material. Here, we tried to achieve a more detailed understanding of the microscopic structure of defects in $\mathrm{MgB}_{2}$. In particular, Raman spectroscopy results showed nicely the effect of defect centers on electron-phonon interaction deviated $E_{2 g}$ mode, which suppressed by increasing defect concentrations. By EPR, we clearly distinguished the $\mathrm{sp}^{2}$ and $\mathrm{sp}^{3}$ hybridization states and clarified that bonding is $\mathrm{sp}^{3}$-like. Distinguishing the $\mathrm{Mg}$ vacancies and $\mathrm{C}$ radicals (dangling bonds) via their $g$-values was one of the other advantages of using EPR spectroscopy. It is also worth mentioning that in this work it was shown that EPR spectroscopy is an effective tool to understand the critical temperature in superconducting materials. EPR showed suppression of $T_{\mathrm{c}}$, which is consistent with present Raman results. Hence, in literature it has been frequently reported that any kind of defects can cause lattice distortion and this distortion is responsible for the decrease in $T_{\mathrm{c}}{ }^{41,43}$

\section{ACKNOWLEDGMENTS}

This work was financially supported by the Deutsche Forschungsgemeinschaft (DFG) with the Grant No. ER 662/ 1-2 and the Koç University Graduate Program. Authors associated with Koç University would like to thank Dr. Bariş Yagci and Koç University Surface Science and Technology Center (KUYTAM) for their support.

${ }^{1}$ C. Buzea and T. Yamashita, Supercond. Sci. Technol. 14, R115 (2001).

${ }^{2}$ J. Nagamatsu, N. Nakagawa, T. Muranaka, Y. Zenitani, and J. Akimitsu, Nature 410, 63 (2001).

${ }^{3}$ S. K. Chen, K. Y. Tan, A. S. Halim, X. Xu, K. S. B. De Silva, W. K. Yeoh, S. X. Dou, A. Kursumovic, and J. L. MacManus-Driscoll, Supercond. Sci. Technol. 26, 125018 (2013).

${ }^{4}$ D. Patel, M. S. Al Hossain, A. Motaman, S. Barua, M. Shahabuddin, and J. H. Kim, Cryogenics 63, 160-165 (2014).

${ }^{5}$ T. Baig, Z. Yao, D. Doll, M. Tomsic, and M. Martens, Supercond. Sci. Technol. 27, 125012 (2014).

${ }^{6}$ J. Ling, J. Voccio, S. Hahn, Y. Kim, J. Song, J. Bascunan, and Y. Iwasa, IEEE Trans. Appl. Supercond. 24, 4400805 (2014).

${ }^{7}$ T. A. Prikhna, M. Eisterer, H. W. Weber, W. Gawalek, V. V. Kovylaev, M. V. Karpets, T. V. Basyuk, and V. E. Moshchil, Supercond. Sci. Technol. 27, 044013 (2014).

${ }^{8}$ A. Patel, S. C. Hopkins, G. Giunchi, A. F. Albisetti, Y. H. Shi, R. Palka, D. A. Cardwell, and B. A. Glowacki, IEEE Trans. Appl. Supercond. 23, 6800604 (2013).

${ }^{9}$ H. Fang, P. Gijavanekar, Y. X. Zhou, P. T. Putman, and K. Salama, IEEE Trans. Appl. Supercond. 15, 3200 (2005).

${ }^{10}$ L. Trevisani, M. Fabbri, and F. Negrini, Cryogenics 47, 113-120 (2007).

${ }^{11}$ M. Maeda, J. H. Kim, and S. X. Dou, "Structural characteristic and superconducting performance of $\mathrm{MgB}_{2}$ fabricated by $\mathrm{Mg}$ diffusion process," in Superconductors - Properties, Technology, and Applications (Intech, 2012).

${ }^{12}$ S. Sanz, T. Arlaban, R. Manzanas, M. Tropeano, R. Funke, P. Kovac, Y. Yang, H. Neumann, and B. Mondesert, J. Phys.: Conf. Ser. 507, 032040 (2014). 
${ }^{13}$ T. Shintomi, T. Asami, G. Suzuki, N. Ota, T. Takao, Y. Makida, T. Hamajima, M. Tsuda, D. Miyagi, M. Kajiwara, and J. Hirose, IEEE Trans. Appl. Supercond. 23, 5700304 (2013).

${ }^{14}$ A. Ballarino, Supercond. Sci. Technol. 27, 044024 (2014).

${ }^{15}$ Y. Hishinuma, S. Yamada, A. Sagara, A. Kikuchi, and T. Takeuchi, Int. At. Energy Agency 43, 419 (2010).

${ }^{16}$ A. L. Ivanovskii, Phys. Solid State 45, 1829 (2003).

${ }^{17}$ I. K. Yanson and Y. G. Naidyuk, Low Temp. Phys. 30, 261 (2004).

${ }^{18}$ C. D. Wang, Y. W. Ma, X. P. Zhang, Z. S. Gao, D. L. Wang, C. Yao, C. L. Wang, H. Oguro, S. Awaji, and K. Watanabe, Supercond. Sci. Technol. 25, 075010 (2012).

${ }^{19}$ M. Maeda, J. H. Kim, Y. Zhao, Y.-U. Heo, K. Takase, Y. Kubota, C. Moriyoshi, F. Yoshida, Y. Kuroiwa, and A. S. X. Dou, J. Appl. Phys. 109, 023904 (2011).

${ }^{20}$ G. Bovone, M. Vignolo, C. Bernini, S. Kawale, and A. S. Siri, Supercond. Sci. Technol. 27, 022001 (2014).

${ }^{21}$ S. K. Chen, K. A. Yates, M. G. Blamire, and J. L. MacManus-Driscoll, Supercond. Sci. Technol. 18, 1473 (2005).

${ }^{22}$ A. Bateni, S. Repp, R. Thomann, S. Acar, E. Erdem, and M. Somer, Appl. Phys. Lett. 105, 202605 (2014).

${ }^{23}$ A. K. Bengtson, C. W. Bark, J. Giencke, W. Dai, X. Xi, C.-B. Eom, and D. Morgan, J. Appl. Phys. 107, 023902 (2010).

${ }^{24}$ A. Vajpayee, R. Jha, A. K. Srivastava, H. Kishan, M. Tropeano, C. Ferdeghini, and V. P. S. Awana, Supercond. Sci. Technol. 24, 045013 (2011).

${ }^{25}$ A. Vajpayee, V. P. S. Awana, S. Yu, G. L. Bhalla, and H. Kishan, Physica C 470, S653 (2010).

${ }^{26}$ V. P. S. Awana, M. Isobe, K. P. Singh, E. Takayama-Muromachi, and H. Kishan, Supercond. Sci. Technol. 19, 551 (2006).

${ }^{27}$ A. Bharathi, S. J. Balaselvi, S. Kalavathi, G. L. N. Reddy, V. S. Sastry, Y. Hariharan, and T. S. Radhakrishnan, Physica C 370, 211-218 (2002).

${ }^{28}$ W. Gruner, M. Herrmann, A. Nilsson, H. Hermann, W. Häßler, and B. Holzapfel, Supercond. Sci. Technol. 20, 601 (2007).

${ }^{29}$ E. Cappelluti, Phys. Rev. B 73, 140505 (2006).

${ }^{30}$ G. M. Zhao, New J. Phys. 4, 3.1 (2002).

${ }^{31}$ J. A. Alarco, A. Chou, P. C. Talbot, and I. D. R. Mackinnon, Phys. Chem. Chem. Phys. 16, 24443 (2014).

${ }^{32}$ N. D. Markovskiy, J. A. Munoz, M. S. Lucas, C. W. Li, O. Delaire, M. B. Stone, D. L. Abernathy, and B. Fultz, Phys. Rev. B 83, 174301 (2011).

${ }^{33}$ Z. C. Tao and M. Singh, Phys. Lett. A 154, 298 (1991).

${ }^{34}$ J. H. Kim, S. Zhou, M. S. A. Hossain, A. V. Pan, and S. X. Dou, Appl. Phys. Lett. 89, 142505 (2006).

${ }^{35}$ R. Flukiger, H. L. Suo, N. Musolino, C. Beneduce, P. Toulemonde, and P. Lezza, Physica C 385, 286 (2003).

${ }^{36}$ W. Mickelson, J. Cumings, W. Han, and A. Zettl, Phys. Rev. B 65, 052505 (2002).
${ }^{37}$ Y. Zhao, Y. Feng, T. M. Shen, G. Li, Y. Yang, and C. H. Cheng, J. Appl. Phys. 100, 123902 (2006).

${ }^{38}$ M. Mudgel, L. S. S. Chandra, V. Ganesan, G. L. Bhalla, H. Kishan, and V. P. S. Awana, J. Appl. Phys. 106, 033904 (2009).

${ }^{39}$ M. Herrmann, W. Häßler, C. Mickel, W. Gruner, B. Holzapfel, and L. Schultz, Supercond. Sci. Technol. 20, 1108 (2007).

${ }^{40}$ M. Avdeev, J. D. Jorgensen, R. A. Ribeiro, S. L. Bud'ko, and P. C. Canfield, Physica C 387, 301 (2003).

${ }^{41}$ A. Bharathi, Y. Hariharan, J. Balaselvi, and C. S. Sundar, Sadhana-Acad. Proc. Eng. Sci. 28, 263 (2003).

${ }^{42}$ A. Motaman, M. S. A. Hossain, X. Xu, K. W. See, K. C. Chung, and S. X. Dou, Supercond. Sci. Technol. 26, 085013 (2013).

${ }^{43}$ P. Parisiades, E. Liarokapis, N. D. Zhigadlo, S. Katrych, and J. Karpinski, J. Supercond. Novel Magn. 22, 169 (2009).

${ }^{44}$ K. P. Bohnen, R. Heid, and B. Renker, Phys. Rev. Lett. 86, 5771 (2001).

${ }^{45}$ A. F. Goncharov, V. V. Struzhkin, E. Gregoryanz, J. Z. Hu, R. J. Hemley, H. K. Mao, G. Lapertot, S. L. Bud'ko, and P. C. Canfield, Phys. Rev. B 64, 100509 (2001).

${ }^{46}$ W. X. Li, Y. Li, R. H. Chen, R. Zeng, S. X. Dou, M. Y. Zhu, and H. M. Jin, Phys. Rev. B 77, 094517 (2008).

${ }^{47}$ O. Chauvet, L. Zuppiroli, and I. Solomon, Mater. Sci. Eng. B 11, 303 (1992).

${ }^{48}$ G. K. Walters and T. L. Estle, J. Appl. Phys. 32, 1854 (1961).

${ }^{49}$ M. R. Mucalo, D. G. McGavin, and N. B. Milestone, J. Mater. Sci. 32, 3271 (1997).

${ }^{50} \mathrm{G}$. Jeschke, M. Kroschel, and M. Jansen, J. Non-Cryst. Solids 260, 216-227 (1999).

${ }^{51}$ B. L. V. Prasad, H. Sato, T. Enoki, Y. Hishiyama, Y. Kaburagi, A. M. Rao, K. Oshida, and M. Endo, Phys. Rev. B 62, 11209-11218 (2000).

${ }^{52}$ F. Berger, A. Muller, F. Aldinger, and K. Muller, Z. Anorg. Allg. Chem. 631, 355 (2005).

${ }^{53}$ D. Odkhuu, D. Shin, R. S. Ruoff, and N. Park, Sci. Rep. 3, 3276 (2013).

${ }^{54}$ G. R. Eaton, S. S. Eaton, D. P. Barr, and R. T. Weber, Quantitative EPR (Springer, Berlin, 2010).

${ }^{55}$ S. K. S. Parashar, B. S. Murty, S. Repp, S. Weber, and E. Erdem, J. Appl. Phys. 111, 113712 (2012)

${ }^{56}$ A. V. Pogrebnyakov, X. X. Xi, J. M. Redwing, V. Vaithyanathan, D. G. Schlom, A. Soukiassian, S. B. Mi, C. L. Jia, J. E. Giencke, C. B. Eom, J. Chen, Y. F. Hu, Y. Cui, and Q. Li, Appl. Phys. Lett. 85, 2017 (2004).

${ }^{57}$ T. Prozorov, R. Prozorov, A. Snezhko, and K. S. Suslick, Appl. Phys. Lett. 83, 2019 (2003).

${ }^{58}$ F. Simon, A. Janossy, T. Feher, F. Muranyi, S. Garaj, L. Forro, C. Petrovic, S. L. Bud'ko, G. Lapertot, V. G. Kogan, and P. C. Canfield, Phys. Rev. Lett. 87, 047002 (2001).

${ }^{59}$ I. A. Garifullin, J. Low Temp. Phys. 178, 243 (2015).

${ }^{60}$ N. Ojha, V. K. Malik, R. Singla, C. Bernhard, and G. D. Varma, Supercond. Sci. Technol. 23, 045005 (2010). 